\title{
Percepción de maestras sobre el acompañamiento fonoaudiológico en un centro de desarrollo infantil en Colombia
}

\section{Perception of teachers about speech therapy accompanying in a child development center in Colombia}

Jenny N. Torres

Universidad del Valle

Natalia Sáenz

Universidad del Valle

María Reyes

Universidad del Valle

Laura Narváez

Universidad del Valle

Contacto con el autor:

Jenny Natalia Torres

Cali - Colombia

Correo-e:

Jenny.natalia.torres@correounivalle.edu.co
RESUMEN

Este trabajo estudió la percepción de maestras beneficiarias de los proyectos de práctica profesional de Fonoaudiología de la Universidad del Valle, respecto al aporte de su acción profesional en la promoción del desarrollo de los niños en el marco de la Política Pública "de Cero a Siempre". La investigación se ejecutó con métodos cualitativos de tipo interpretativo y de corte transversal, bajo el diseño de estudio de caso. La exploración permitió concluir que el acompañamiento fonoaudiológico: i) es más efectivo cuando se reconoce y valora los saberes y requerimientos de los beneficiarios, ii) enriquece los procesos de desarrollo de niños y niñas en general y no sólo a aquellos con patologías del lenguaje o discapacidad y iii) potencia el objeto de la política pública de atención a la primera infancia en Colombia. Adicionalmente, valida la acción fonoaudiológica desde una propuesta situada y bajo el enfoque comunitario.

Palabras clave: primera infancia, comunicación y desarrollo, cualificación de docente de preescolar, desarrollo infantil.

\begin{abstract}
This study was focused on the perception of teachers who participated as beneficiaries on the professional practice in speech therapy implemented by the Universidad del Valle. The main focus was on how participants' professional intervention promoted the development of children, within the framework of the Public Policy "de Cero a Siempre". The practice was developed at Universidad del Valle from August 2013 to June 2016. The research was conducted using interpretative qualitative methods and was cross-sectional (case-study design). The study concluded that: i) success of the speech therapy intervention is related to the recognition and evaluation of the beneficiaries' requirements ii) the speech therapy intervention improves development processes of all children, not only of children with language pathologies or disabilities, iii) the intervention fosters the Public Policy of attention to Early Childhood in Colombia. Finally, the observed practices validate speech therapy action from a community approach.
\end{abstract}

Keywords: early childhood, communication and development, pre-school teacher qualification, child development.

Recibido: $17 / 10 / 2017$

Aceptado: 10/05/2018 


\section{Introducción}

En Colombia desde el año 2010, se ejecutan acciones para la atención a la Primera Infancia en el marco de la Política Pública "de Cero a Siempre". En ese marco, se han dispuesto servicios de educación inicial bajo tres modalidades -familiar, comunitaria e institucional- que posibilitan la protección y el desarrollo infantil (Instituto Colombiano de Bienestar Familiar, 2012). Lamentablemente, en ese escenario no se ha contemplado la acción fonoaudiológica como un recurso fundamental para el acompañamiento y la mediación de los entornos de educación inicial. Hasta el año 2012, se consideraba que el profesional fonoaudiólogo presentaba un perfil que le permitía asumir tareas pedagógicas. Sin embargo, posteriormente esta idea se diluyó en los anexos técnicos de contratación del talento humano (de la Fuente, 2012).

Al realizar un análisis de la investigación disciplinar en Colombia se observa que la población con mayores porcentajes de investigación en Fonoaudiología es la infantil con una tasa de un $70 \%$. Sin embargo, la mayoría de estas investigaciones se orientan a la definición de diagnósticos en el área de lenguaje (Montes, Quessep, Redondo, \& Lastre, 2017), lo que repercute en una patologización, casi generalizada, de la infancia. Lo anterior, se opone a las políticas de reconocimiento de los desempeños en la diversidad en los modelos de desarrollo infantil.

También, es importante señalar que a nivel nacional e internacional existe una tendencia a investigar sobre la intervención de procesos patológicos del desarrollo infantil. Se centralizan en aspectos formales del lenguaje, en roles asistenciales de evaluación y en el establecimiento de un diagnóstico (Lizarazo \& Fique, 2013; Martínez, 2007). A su vez, se sustentan en el análisis de implementaciones de escalas y en la interpretación de test de desarrollo del lenguaje. Algunos de ellos son: Language Exposure Assessment Tool (LEAT) y el Achievement of the First Milestone of Oral Language (FMOL), (DeAnda, Bosch, Poulin-Dubois, Zesiger, \& Friend, 2016; Muñoz-Caicedo, Zapata-Ossa, PérezTenorio, \& Illera, 2012; Pianesi et al., 2016).

En este contexto, Pava-Ripoll, Payán, \& Reyes (2011) analizaron la tendencia investigativa de los trabajos de grado del programa académico de Fonoaudiología más importantes del suroccidente colombiano, encontrando que la infancia y la primera infancia son los ciclos vitales donde se han realizado la mayoría de las investigaciones $(13 \%$ de 98 documentos revisados). Estos autores mostraron que dentro del componente de intervención las acciones de habilitación/rehabilitación (41\%) han prevalecido por sobre las de promoción y prevención (33\%). El modelo rehabilitador aparece con un predominio de un 53\%, evidenciándose un mayor acercamiento hacia los modelos sociales a partir del año 2003.

Adicionalmente, Duarte (2011) presentó el estado del arte de investigaciones en Fonoaudiología acerca de la atención y el desarrollo de la primera infancia en diferentes municipios colombianos entre los años 1994 y 2005. Afirmó que los estudios de infancia se correlacionan directamente en contextos de educación formal con los grados de transición y primero de primaria, es decir, en niños y niñas de 4 a 6 años. Sin embargo, reporta que existen escasas investigaciones cuyo objeto de estudio corresponde a la familia y a otros agentes socializadores, como 
madres comunitarias y cuidadores.

Teniendo en cuenta dicho panorama, es necesario la re-categorización de la Fonoaudiología, y con ello, el establecimiento de una nueva concepción y puesta en marcha en un contexto latinoamericano particular. Este contexto requiere el reconocimiento de características históricas y sociales que se traducen en la dinamización del objeto de estudio disciplinar, con el fin de comprender la comunicación, el lenguaje y la diversidad dentro de un proyecto de sociedad. De tal manera, la Fonoaudiología adquiriría sentido en el marco de las realidades sociales y comunitarias actuales de los países latinoamericanos.

Según Yáñez (2013), lo anterior exige asumir el escenario de lo social en donde lo disciplinar existe como un ser en sí y ser para el mundo, es decir, como proyecto histórico-procesual, teórico-metodológico, ético-político y crítico-ideológico. Esto implica que las intervenciones deben ser fundamentadas, transformadoras y reconocedoras de la diversidad en donde se toman posturas rigurosas en un intento de conversar con un mundo social complejo, polisémico y multidimensional. Es por ello, que es indispensable la sistematización de experiencias que apuntan a la problematización disciplinar de la Fonoaudiología en contextos reales, pues, el lenguaje, la comunicación y la inclusión adquieren sentido en la acción situada. Desde esta perspectiva, el desarrollo se entiende como:

organizador de los procesos educativos de la primera infancia, en el que cobra mucha importancia la calidad de las interacciones que establecen las maestras, maestros y los agentes educativos con las niñas y los niños, así como la calidad de los ambientes enriquecidos en donde estas interacciones tienen lugar, requiriendo de prácticas pedagógicas intencionadas (Téllez, Camargo, Reyes, \& Suárez, 2014, p. 71).

Esta concepción de desarrollo exige proponer interacciones significativas y relevantes, ambientes enriquecedores y adecuados para el desarrollo, junto con la observación y escucha atenta. También el establecimiento debe propiciar redes con la familia como procesos clave que otorgan sentido a la educación inicial y que pueden ser acompañados desde la acción fonoaudiológica.

En las interacciones significativas y relevantes es importante que los niños y niñas sean escuchados y considerados respecto a sus ritmos de desarrollo, intereses, emociones y formas de ser. Todo lo anterior posibilitará que niños y niñas se sientan reconocidos, queridos, acogidos y valorados. Es por esto que las relaciones que se crean deben contribuir a que los niños y niñas accedan a los significados de la cultura y la manera en que se inscriben en la realidad social (Camargo, Reyes, \& Suárez, 2014a). Adicionalmente, las relaciones deben estar mediadas por las actividades rectoras de la primera infancia (juego, arte, literatura y exploración del medio) promoviendo la creatividad, el aprendizaje y el desarrollo de las niñas y los niños (Camargo, Reyes, \& Suárez, 2014b).

Los ambientes enriquecedores y propicios para el desarrollo, corresponden a "los ámbitos espaciales, temporales y relacionales en los cuales cada niña o niño desarrolla sus propias experiencias de vida" (Camargo et al., 2014a, p. 72), los que incluyen a "los 
sujetos que intervienen, a las relaciones que allí se tejen y a la manera como se apropian y usan los espacios y materiales disponibles para provocar experiencias pedagógicas significativas" (Camargo et al., 2014a, p. 72-73). Estos ambientes se caracterizan por

la libertad de expresión de las emociones, de los sentimientos, de las preguntas e inquietudes y por las interacciones naturales, en tanto son condiciones que enriquecen y potencian el desarrollo infantil. Asimismo, la calidez del ambiente educativo, el afecto, la confianza y la seguridad son fundamentales para la promoción del desarrollo infantil (Camargo et al., 2014a, p. 73).

La observación y escucha atenta son acciones que "permiten dar un primer paso para descifrar las formas propias de ser de las niñas y de los niños, descubrir que en un grupo puede haber intereses comunes o miedos y gustos particulares" (Téllez et al., 2014, p. 24). Por lo tanto, a través de estas acciones se realiza un seguimiento y reconocimiento de los desempeños para brindar un mejor acompañamiento al desarrollo integral infantil.

La creación de redes con la familia se establece como parte del proceso educativo en la primera infancia, con el fin de crear estrategias conjuntas que favorezcan el desarrollo de las niñas y los niños. También se busca la participación activa de las familias en el intercambio de saberes, opiniones, dudas, dificultades, logros, entre otros aspectos, con maestras y maestros (Camargo et al., 2014b).

Por otra parte, existen otros aspectos que son valiosos en la construcción del desarrollo integral que atienden a dimensiones más individuales. Los niños y las niñas "utilizan sus capacidades para iniciar un proceso creciente de integración a la vida social como protagonistas de su propio desarrollo" (Instituto Colombiano de Bienestar Familiar, 2012, p. 19). Al respecto, el Ministerio de Educación Nacional menciona la participación y la autonomía como constituyentes de la educación inicial para favorecer el desarrollo infantil (Camargo et al., 2014b, p. 77).

La participación se trata de un derecho consagrado en el artículo 12 de la declaración de los derechos del niño que "consiente a los niños en desempeñar en su propia vida un papel protagónico, en vez de ser simplemente beneficiarios pasivos del cuidado y protección de los adultos" (Lansdown, 2005 , p. 1). Esto implica que son capaces de manifestar su opinión y tienen derecho a expresarla libremente, a ser escuchados en todos los asuntos que les afectan $y$ a que sus opiniones sean consideradas seriamente. La participación infantil es un proceso fundamental en la formación de sujetos críticos, reflexivos, reconocedores, propositivos, innovadores y transformadores. En este aspecto también interviene la visión de los adultos al reconocer a los niños y niñas como interlocutores válidos con derecho a expresar sus opiniones y con capacidad para decidir (Téllez et al., 2014).

La autonomía es entendida como un proceso que les permite a niños y niñas el desenvolvimiento libre, espontáneo y seguro en los entornos donde transcurre la vida, que implica actuar, proponer, resolver, ejecutar y elegir. La autonomía permite "dejar de ser un ser absolutamente dependiente, para convertirse en un sujeto ejecutor. Es el 
momento en que los saberes y haceres, transformados completamente en poder hacer, necesitan el tiempo y los grados de libertad para actuar" (Puche, Orozco, Orozco, \& Correa, 2009, p. 43).

El reconocimiento de la acción disciplinar en Fonoaudiología en el marco de la política pública requiere considerar el desarrollo infantil, situándose en un paradigma que valora aspectos de la diversidad infantil en sus contextos reales. Este paradigma es contrario a la mirada hegemónica de la Fonoaudiología que se basa en análisis de estructuras que suelen ser incompletos.

Desde el año 1987 la Universidad del Valle, perteneciente al Suroccidente Colombiano ha acompañado a niños, niñas, familias, madres comunitarias, maestras ${ }^{1}$, maestros, directivos en entornos de educación inicial ubicados en las comunas 18 y 20 de la ciudad de Cali. El acompañamiento busca potenciar interacciones asertivas en el plano verbal y no verbal desde la Atención Primaria en Salud, la cual, fue enriquecida desde el año 2010 con las propuestas de Rehabilitación Basada en Comunidad (RBC), que se basa en un enfoque comunitario.

Desde esta perspectiva, se recuperan las voces de quienes participaron como beneficiarios del acompañamiento fonoaudiológico, de tal forma que se valida la efectividad del acompañamiento en función del desarrollo humano propiamente dicho (Sen, 2000), en el marco de las posibilidades que otorga el sistema actual. Es por ello, que se reconoce

${ }^{1}$ Para efectos de comprensión de este documento se utiliza este referente denotando maestras y maestros. Se utiliza en femenino reconociendo que la mayoría de personas que ejercen esta labor en la infancia son mujeres. que las maestras han jugado un rol central en el acompañamiento desde una acción participativa y dialógica, convirtiendo la interacción en un aspecto fundamental que complementa los ámbitos biológico, psicológico, cultural y social de sí mismas y de los niños y las niñas con que comparten cotidianamente.

\section{Objetivos}

El objetivo general de este estudio fue develar, desde la voz de las maestras, los aportes del acompañamiento fonoaudiológico al desarrollo de los niños y las niñas de 0 a 5 años, quienes presentan condiciones de diversidad propias de la comunidad en la que se encuentran.

Los objetivos específicos fueron: 1. Explorar las percepciones de las maestras respecto al ejercicio de la práctica profesional en Fonoaudiología en el Centro de Desarrollo Infantil; 2. Identificar respuestas de las acciones fonoaudiológicas en el marco de la propuesta de desarrollo dispuesta en los documentos del Instituto Colombiano de Bienestar Familiar y el Ministerio de Educación, y 3. Generar reflexiones frente a la necesidad de la dinamización disciplinar en Fonoaudiología ante la necesidad de atención a entornos de educación inicial en Colombia.

\section{Método}

El estudio se enmarcó en la experiencia de la práctica profesional de la universidad del Valle. Esta experiencia consistió en el acompañamiento de estudiantes y profesores de Fonoaudiología a 
maestras que se encontraban a cargo de grupos de niños y niñas organizados por los siguientes bloques de edad: 2 a 3 años, 3 a 4 años y 4 a 5 años. Se efectuó, desde agosto del año 2013 hasta diciembre del año 2016, en entornos de educación inicial del ICBF en un CDI ubicado en el barrio las Palmas de la Comuna 18.

La presente investigación se realizó bajo el diseño de estudio de caso que incluyó una muestra intencional cubriendo la totalidad de la población conformada por 7 maestras. Los criterios de inclusión fueron los siguientes: a) maestras con vinculación laboral en el CDI de al menos 1 año y que hayan recibido acompañamiento directo por parte estudiantes de Fonoaudiología, b) que tuvieran a cargo un grupo de niños entre 0 a 5 años y c) que accedieran a participar de manera voluntaria firmando como soporte el consentimiento informado.

Se obtuvo la información mediante una entrevista ${ }^{2}$ en profundidad con características de guiada, directa e individual (Taylor \& Bogdan, 1994). Las entrevistas fueron grabadas y posteriormente transcritas. La información se organizó asignando códigos alfanuméricos de registro a la transcripción

\footnotetext{
${ }^{2}$ La pauta entrevista contó con las siguientes preguntas: 1 . ¿Hace cuánto trabaja en el CDI?, 2. ¿Qué grupos ha asumido desde su ingreso al CDI?, 3. ¿Ha recibido acompañamiento por parte de Programa Académico de Fonoaudiología de la Universidad del Valle? ¿Cómo ha sido ese acompañamiento?, 4. ¿Qué hacen las personas que vienen del PAF (Los profesores y los estudiantes)?, 5. ¿Siente que ha aprendido algo de ellos? ¿Qué cosas?, 6. ¿Siente que usted les ha podido enseñar algo a ellos? ¿Qué cosas? ¿Le han manifestado lo que han aprendido de usted?, 7. ¿Qué tipo de propuestas han tenido para los niños y niñas?, 8. ¿Siente que lo que hacen con los niños y niñas es importante? ¿Por qué?, 9. ¿Aparte de hacer trabajo con usted y los niños y niñas han realizado actividades con otras personas? (familia, comunidad $u$ otros), 10. ¿Se ha sentido escuchado por las personas que vienen del PAF para el diseño de sus propuestas?, 11. ¿Cómo es la relación de las personas que vienen del PAF con los niños y niñas? ¿Respecto a esa relación usted les ha podido enseñar algo o usted ha podido aprender algo de ellos?
}

de cada persona entrevistada. Así, se evitó correlaciones de nombre con el fin de proteger la identidad de los participantes.

Se categorizó el contenido de las respuestas por agrupación de enunciados que concentraron ideas, conceptos o temas similares (Rubin \& Rubin, 1995). En una matriz de Excel se identificó cada categoría de análisis y se definieron criterios de agrupación por colores para cada una. A partir de este proceso surgieron las siguientes categorías preliminares: participación, autonomía, interacciones significativas y relevantes, construcción de ambientes que potencian el aprendizaje y mejores relaciones sociales, trabajo en red con familias, capacidad de observación que permite reconocer los ritmos, intereses, emociones y formas de ser de cada niño. También, se consignó una categoría denominada diversidad, que emergió a partir de las respuestas de las participantes en la investigación.

\section{Resultados}

A continuación se exponen los resultados para cada una de las categorías sustraídas de los referentes técnicos de educación inicial (Caro, Chávez, \& Vásquez, 2014). Para su comprensión, es importante señalar que se describirán desde la definición teórica de tal forma que se identifique la respuesta de las acciones fonoaudiológicas frente al desarrollo infantil en el marco de los referentes técnicos a nivel nacional. Finalmente, se presentan algunas reflexiones frente a la dinamización disciplinar en el marco del acompañamiento fonoaudiológico que establece participar en entornos de educación inicial en Colombia. 


\section{Interacciones significativas y relevantes}

En el contexto de las interacciones de niños y niñas con sus pares y con los adultos se construyen relaciones significativas que se caracterizan porque los niños sean escuchados y considerados en sus ritmos de desarrollo, intereses, emociones y formas de ser (Camargo et al., 2014a). Dichas relaciones potencian el desarrollo de los niños y las niñas, ya que les posibilita aprender del mundo, de los adultos que les rodean y de ellos mismos.

En relación a esto, las maestras refirieron que la base de una interacción significativa debe estar mediada por el respeto, ya que propicia escuchar al otro, reconocerlo y otorgarle un lugar, facilitando que se tejan relaciones basadas en la tolerancia y en el respeto. Estas relaciones se construyen de manera recíproca, pues según las maestras, las interacciones significativas involucran un proceso de aprendizaje bidireccional, en el que la actitud que expresan los adultos mediante el lenguaje verbal y no verbal juega un papel importante en cómo se relacionan con otros, creando relaciones empáticas que propicien la construcción de ambientes pedagógicos.

En relación al acompañamiento fonoaudiológico, algunas maestras mencionaron que este les ha ayudado a comprender que existen múltiples manifestaciones no verbales que los niños y las niñas utilizan para comunicarse. Por ello, se han preocupado por indagar qué quieren expresar mediante sus actos no verbales otorgándole una intencionalidad comunicativa a dichos actos. También, refirieron que a partir del acompañamiento han podido enriquecer la forma en que comprenden en la acción cotidiana las interacciones significativas y relevantes, pues, en su ejercicio diario han integrado saberes $\mathrm{y}$ actitudes para reconocer $\mathrm{y}$ validar aquello que niñas y niños expresan desde sus posibilidades verbales y no verbales, reconociéndolos como sujetos de derechos e interlocutores válidos.

Asimismo, la comprensión de las diferentes formas no verbales que los niños empleaban para expresar sus emociones, les permitió a las maestras asignar un sentido a este tipo de expresiones, como adultos que orientan y acompañan, pueden dar una respuesta que les permite apoyar a los infantes en el desarrollo de recursos comunicativos que les conduzcan a lograr una mayor autonomía en sus relaciones sociales mediante la adopción de formas comunicativas para manifestar sus emociones de manera asertiva.

"Sí, sí me ayudó bastante porque muchos de los niños se comunicaban a través del llanto y yo no podía entender qué era lo que de pronto estaba pasando y con la explicación que ellos me daban pues me acerqué a ellos, a escucharlos y si pude observar que sí tuvieron una ayuda de ellos, de parte de ellos." (Fragmento de entrevista de la maestra \#7)

De acuerdo con la (Comisión Intersectorial para la Atención de la Primera Infancia, 2012a) otorgarles un lugar privilegiado a niñas y niños dentro de las interacciones, da cuenta del reconocimiento de su capacidad participativa y construcción ciudadana. Por ende se los concibe como sujetos de derechos e interlocutores válidos. En este sentido, las maestras expresan que, con el acompañamiento fonoaudiológico, han consolidado herramientas 
como la observación y la escucha frente a los niños y las niñas, ofreciendo apoyos basados en canciones y preguntas intencionadas que los invitan a expresar sus ideas. Adicionalmente, las maestras reconocen el diálogo como un apoyo para fomentar la participación, además de reconocer a los niños y las niñas, como sujetos con capacidad para participar de ese diálogo.

"Por lo menos con la niña que casi no hablaba, observarla, mirarle que dice y hablarle. Por lo menos Andrea (Profesora de la práctica en fonoaudiología) me decía que le hablara mucho a Tania, que le cantara y ella empezó a soltarse y a soltarse hasta que ya no para de hablar esa niña." (Fragmento de entrevista de la maestra \# 2)

\section{Construcción de ambientes enriquecidos y propicios para el desarrollo}

Los ambientes enriquecedores y propicios para el desarrollo refieren a "los ámbitos espaciales, temporales y relacionales en los cuales cada niña o niño desarrolla sus propias experiencias de vida" (Camargo et al., 2014a). De igual forma, los ambientes incluyen a "los sujetos que intervienen, a las relaciones que allí se tejen y a la manera como se apropian y usan los espacios y materiales disponibles para provocar experiencias pedagógicas significativas" (Camargo et al., 2014a).

Estos ambientes deben caracterizarse por la libertad de expresión de las emociones, sentimientos, preguntas e inquietudes y por las interacciones naturales, en tanto son condiciones que enriquecen y potencian el desarrollo infantil.
Asimismo, la calidez del ambiente educativo, el afecto, la confianza y la seguridad son fundamentales para la promoción del desarrollo infantil (Camargo et al., 2014a).

Para las maestras los ambientes enriquecidos deben construirse en torno a las actividades rectoras de la primera infancia como son: el juego, el arte, la literatura y la exploración del medio. Una de las actividades que más destacan para generar un ambiente enriquecedor es el juego, porque permite promover aprendizajes tanto individuales como colectivos. Además, potencia el desarrollo comunicativo, ya que pueden expresar sus deseos y sus emociones. En este sentido, las maestras valoraron los ambientes propiciados por los estudiantes de fonoaudiología.

"Me pareció chévere porque llegaban y les hacían las actividades, les traían... ayudaban mucho. Les hacían cuentos, les hacían juegos, actividades de texturas, traían muchas actividades sensoriales, de sonidos, con tarros, eso a los niños les gustaba mucho." (Fragmento de entrevista de la maestra \# 2)

Otra actividad valorada por las maestras es la lectura de cuentos porque les permitió reconocer que leer va más allá del código escrito, al poner en juego otro tipo de lecturas en relación con la actitud corporal, los sentimientos y los significados que se construyen. También, reconocen que la lectura en la primera infancia requiere de un mediador que dé vida al texto a través de su voz. 
"Con la lectura de cuentos he aprendido a manejar esas diferentes voces, tener en cuenta las representaciones o el rol de cada personaje; por ejemplo, si hay un personaje que está bravo entonces hay que hacer la voz de una persona que realmente está enojada. Si es una persona que está llorando, hacer los sonidos, utilizar como esas voces, esas pausas, preguntarles a los niños" (fragmento de entrevista de la maestra \# 4)

Sin embargo, más allá del ambiente físico y material, los ambientes enriquecidos deben ser afectivos para generar calidez, afecto, confianza y seguridad, permitiendo la construcción de vínculos de apego seguros. En relación con esto, las maestras destacaron que el acompañamiento fonoaudiológico les ha permitido reconocer que un ambiente afectivo es importante en la medida en que el niño sienta seguridad y confianza para expresarse. Lo anterior demuestra que la disposición emocional del adulto es muy importante en la interacción con los niños.

"yo muchas veces me he quejado de que los niños no se escuchan, hablan muy duro, y gritan. Entonces he implementado esas cosas que me dicen es que, si tú quieres que el grupo esté tranquilo, tú también tienes que estar tranquila" (Fragmento de entrevista de la maestra \# 4)

\section{Participación}

El concepto de participación es una construcción cultural a partir de la convivencia con valores y principios éticos. Los niños y las niñas experimentan a través de la participación, la libertad para construir su realidad e interactuar con los y las demás, con los ambientes, con ellas y ellos mismos (Comisión Intersectorial para la Atención de la Primera Infancia, 2012b).

A partir del proceso de participación los niños y las niñas se constituyen como sujetos críticos, reflexivos, reconocedores, propositivos, innovadores y transformadores. A su vez, los adultos los conciben como personas capaces de tomar decisiones y de presentar opiniones respecto a su propia vida, por tanto, tienen derecho a ser consultados sobre las situaciones que les acontecen (López \& Tobón, 2015).

Para las maestras, la participación implica que los niños y las niñas se sientan cómodos, contentos y se muestren atentos durante las actividades individuales y grupales. Además, involucra el espacio que les permita expresar sus opiniones y sentimientos a sus pares, a sus maestras y a otros interlocutores.

"Ellos dicen "yo tengo eso" y yo les preguntó "Miren, ¿qué es esto? ¿Quién tiene esto?" por ejemplo un perro, "¿quién tiene un perro en su casa?", entonces empiezan a hablar y ahí vamos contando el cuento, ellos van interactuando". (Fragmento de entrevista de la maestra \#2)

Las capacidades infantiles para la participación social se determinan en mayor medida por factores como las expectativas de su contexto social y cultural más que por sus características biológicas y psicológicas (Lansdown, 2005). Generalmente, los adultos acostumbran a considerar a los niños como seres inmaduros fisiológica y psicológicamente, por lo que se espera poco de ellos con respecto al resto 
de las personas, restándole importancia a sus propios deseos y sentires.

Las maestras afirmaron que el juego posibilita la creación de un espacio de escucha y potencializa las capacidades y habilidades de las pequeñas y los pequeños, ya que mediante el juego pueden desplegar sus múltiples lenguajes y sus formas de actuar y de crear. Es por esto que un aporte relevante del equipo de estudiantes y profesores de Fonoaudiología, según comentan las maestras, es el incremento de actividades grupales, las cuales fomentan el uso de estrategias comunicativas complejas y promueven el juego como herramienta para el aprendizaje.

\section{Trabajo en red con las familias}

Desde el Ministerio de Educación Nacional se establece que el proceso educativo en la primera infancia debe realizarse en colaboración con las familias. Con esta colaboración se busca la creación de estrategias conjuntas que favorezcan el desarrollo de las niñas y los niños. Además, se incentiva la participación activa de las familias en el intercambio con las maestras de saberes, opiniones, dudas, dificultades y logros (Camargo et al., 2014b). La comunicación continua del centro educativo con las familias es fundamental, ya que ambos espacios pueden aportar información valiosa acerca del desarrollo de los niños y las niñas de manera que se pueda generar un seguimiento.

Desde el Programa Académico de Fonoaudiología de la Universidad del Valle se ha venido trabajando tanto con niños y niñas como con sus maestras dentro de los espacios del CDI. Sin embargo, no todas las maestras refieren tener conocimiento o haber presenciado interacción entre las familias de los niños y niñas con los estudiantes y docentes del programa.

"Por lo menos durante el año pasado, que yo me haya dado cuenta que intervinieron con los agentes externos, no". (Fragmento de entrevista de la maestra \#3).

Por su parte, algunas maestras manifiestan que desde el programa se ha realizado seguimiento con las familias.

"El año pasado citaron a las mamás de los niños, que se les hizo el seguimiento. Se hizo reunión con los papás y con los fonos para darles la estrategia o explicarles el proceso que tenían con las niños."

(Fragmento de entrevista maestra \#5).

Uno de los aportes valorado es la creación de espacios en los cuales se logra compartir experiencias, estrategias de trabajo e inquietudes respecto al desarrollo de los niños y las niñas. Lo anterior indica que la interacción entre el Programa Académico de Fonoaudiología y las familias de los pequeños y pequeñas debe visibilizarse y negociarse con mayor fuerza con las maestras. Ello permitirá potenciar el desarrollo infantil, ya que se crean canales de comunicación más significativos entre maestras, familias, estudiantes y docentes de Fonoaudiología. Así, se dispone de información precisa y consensuada sobre las normas, los retos y apoyos que deben recibir los niños y las niñas. 


\section{Autonomía}

De acuerdo con lo definido en los lineamientos para la atención a la primera infancia, entre el primer y el tercer año de vida, los niños inician un lento y largo trayecto hacia su autonomía. En este proceso es de vital importancia el acompañamiento por parte de los adultos quienes, a partir de su mediación, orientan y brindan un ambiente de confianza que les permite a los niños la creación de nuevas relaciones (López \& Tobón, 2015).

Las maestras reconocen la autonomía como la posibilidad de que niños y niñas comiencen a realizar las actividades sin ayuda de los adultos, como es el caso de alimentarse y relacionarse con sus pares durante las actividades. Para esto, afirman que es importante que se brinden espacios y estrategias que permitan impulsar su propio aprendizaje.

Respecto a los aportes del acompañamiento fonoaudiológico, identifican acciones que contribuyen a la conquista de la autonomía de los niños, entre las que se incluyen espacios de interacción que crean un clima de confianza. La confianza que entregan estos climas permite que los niños se expresen con sus pares y generan interacciones en los que se observa una confianza creciente en la capacidad de los infantes para realizar las actividades de autocuidado.

\section{Observación y escucha atenta}

De acuerdo con (Camargo et al., 2014b) el proceso de seguimiento y observación parte de la comprensión de los niños como seres únicos e irrepetibles. Implica el reconocimiento de la diversidad en el proceso de desarrollo, intereses, capacidades y maneras de aprender, interacciones y necesidades afectivas y de cuidado.

Las maestras reconocen en las expresiones no verbales de los niños una intención comunicativa y en este sentido se adaptan como interlocutores para situarse a su nivel. Además, de reconocer sus diferentes formas de expresión, las maestras reconocen la capacidad que tienen los niños para realizar lecturas del contexto en el que se encuentran y de las personas que son parte del mismo.

La observación es concebida por las maestras como un proceso que requiere involucrarse $y$ participar de la situación en la que se encuentra el niño. Según lo que mencionan, observar está siempre ligado a acompañar.

"En el momento que ellos quieren expresar algo, de pronto por medio de un llanto o alguna tristeza acercarse a ellos, dialogar con ellos y tratar un poco de investigar qué es lo que está sucediendo, de pronto sea en casa o alguna otra situación. Por ejemplo, en el momento que ellos son agresivos por lo menos sentarse con ellos. Primero, observar, primero que todo observar al niño, observar qué comportamiento está realizando y de acuerdo con eso, sentarse con él y dialogar". (Fragmento de entrevista de la maestra \#7)

El proceso de observación realizado por maestras trasciende a la historia de vida de cada niño y niña, las lecturas que realizan son globales a partir de lo cual se evidencia una mirada del desarrollo amplia, en la que se incluyen tanto elementos inherentes a la 
persona como a los elementos presentes en el contexto.

"trabajar con niños pequeños es un voltaje distinto, es desarrollar habilidades, aprenderlos a conocer, leer lenguajes a veces muy no verbales, leer el contexto de la familia". (Fragmento de la maestra \#6)

Con relación al acompañamiento fonoaudiológico, mencionan que los aportes realizados se relacionan con la posibilidad de realizar una observación más puntual de algunos aspectos específicos. Por ejemplo, el conocimiento sobre las etapas del desarrollo motor, que les han permitido entregar apoyos a los niños en la rutina de alimentación para que este proceso sea cada vez más independiente.

"mis niños, no todos comen, entonces toca estarles dando entonces hasta que uno no llega... Y uno "vea coma", entonces ellas decían que era importante ellos mismos cojan la cuchara. Eso lo hago pero hay veces que se me olvida pero luego me acuerdo. Lo del turno también para mí era difícil debido a la actividad que hicieron afuera en el corredor qué hora sentarse ahí, ya me dieron pautas para seguir, como decirle a los niños que esperar el turno porque era dificilísimo y ahora ya no es tan difícil." (Fragmento de entrevista de la maestra \#2)

\section{Diversidad}

En la investigación surgió una categoría emergente que aludía a las reflexiones frente a la diversidad en la infancia que se consolida como una categoría transversal. En este sentido, los niños y las niñas son reconocidos en sus necesidades $y$ capacidades específicas en un contexto determinado. Las maestras identifican a los niños y las niñas como seres singulares que merecen ser reconocidos y que requieren apoyos específicos para su desarrollo. Incluso, señalan que todos los niños y las niñas merecen una vida digna y un contexto que garantice sus derechos. Esta idea incluye a los niños y las niñas con discapacidad.

"Primero, observar, primero que todo observar al niño, observar que comportamiento está realizando y de acuerdo con eso, sentarse con él" (Fragmento de entrevista de la maestra \# 7).

"Pues yo considero y creo que la educación del niño esta como que si, la institucionalidad y también la familia. Por ejemplo, yo tenía un caso de un chico que tenía problemas con la ensalada, entonces yo hablaba con la mamá y ella me decía "no, es que yo no como ensalada" entonces imagínate, el foco a veces o lo que nos traen los niños acá es el reflejo de lo que viven en la familia ... y jugamos con toda la imaginación y con todo el desarrollo del niño y hay cambios, pero entonces el niño también se podría confundir porque de pronto aquí en el CDI ve unas cosas y en la familia ve otras. Es importante un trabajo con los puestos de salud en el sentido, tener un acercamiento a las familias, en este caso, para el manejo de la alimentación y de las demás instituciones que le podrían aportar al niño" (Fragmento de entrevista de la maestra \# 6). 
Es necesario señalar que las propuestas de los proyectos de práctica ejecutados en el CDI se encuentran basadas en la estrategia de Rehabilitación Basada en Comunidad (RBC), las que promueven oportunidades a las personas con discapacidad (Organización Mundial de la Salud, 2009). Para el caso específico de esta investigación, el acompañamiento no sólo ha empoderado a las maestras respecto a la discapacidad, sino que se ha ampliado el espectro hacia otro tipo de poblaciones que requieren apoyo y que son fuente de reflexión en el mundo latinoamericano.

"Cuando ustedes vienen logran ver cosas que uno no ve, por ejemplo que el niño no tiene mucho movimiento, no sé, o no tiene mucha interacción con las demás personas, es muy aislado; a veces uno eso no lo nota tanto o no le presta tanta atención, en cambio ustedes lo pueden notar y pueden generar estrategias o simplemente decirnos, ve profe, es que vemos que, que este niño tiene esto y esto, como lo has visto, el comportamiento, entonces uno ya se genera una inquietud y a partir de eso uno puede como estar más pendiente de ese niño y buscar rutas o apoyos, hablar con el padre de familia a ver qué es lo que está pasando". (Fragmento de entrevista del maestro \# 3).

"Tuve tres niñas en seguimiento, una niña porque ella no pronunciaba nada, no hablaba mucho y otras dos niñas que fueron las gemelas porque ellas se demoraban mucho en el momento de la alimentación y acumulaban la comida en la boca, entonces a ellas se les hizo un seguimiento y se hizo reunión con los papás y con los fonos para darles estrategias y explicarles" (Fragmento de entrevista del maestro \#6).

Las maestras con el acompañamiento han activado rutas para garantizar los derechos de los niños y las niñas a nivel interno y externo. A su vez, han consolidado saberes que les permiten entregar apoyos, en el entorno de educación inicial, que facilitan el desarrollo infantil.

"que tenía problemas para subirse, para tener contacto, le daba miedo, entonces pues sí me reglaron como algunas pautas y el niño la verdad ha mejorado mucho" (Fragmento de entrevista del maestro \#1).

"Por lo menos en las actividades que ellos han hecho me ha parecido chévere y por lo menos el año pasado tuve una niña que no caminaba. Entonces yo le preguntaba a ellas que como hacía. Que hágale masajitos, hágale así y así, y por lo menos en la alimentación, ellas también me decían. Entonces yo les pregunto y yo trato de hacerlo" (Fragmento de entrevista de la maestra \#2).

"Nos han enseñado las diferentes formas de expresión de los niños, la escritura, lo importante que es. Y lo de lo verbal como tal, pues no solamente puedo decir que nos han como enseñado como esas dificultades que los niños tienen, si no como el porqué de las cosas, por ejemplo, que, porque un niño está chupando 
dedo, entonces todo tiene una razón de ser".

(Fragmento de entrevista de la maestra \#4).

Se puede afirmar que las maestras reconocen a los niños y las niñas con discapacidad como un sujeto que es digno y capaz de compartir en el entorno de la educación inicial y que requiere apoyos específicos para propiciar su desarrollo. En este sentido, se identifican como corresponsables con la familia y con otras instituciones y en su rol pedagógico asumen lo que les compete para garantizar los derechos infantiles. Las maestras identifican en todos los niños y las niñas, no sólo en aquellos con discapacidad, la necesidad de ser dinámicas y flexibles, de entender las características, los modos de vida y los contextos. Todo ello permite la configuración de apoyos que respetan la dignificación humana.

\section{Conclusiones}

En cuanto a la percepción que tienen las maestras del acompañamiento, es posible afirmar que reconocen el intercambio de conocimiento con los fonoaudiólogos imprescindible. Ello facilita un proceso de aprendizaje bidireccional para alcanzar un objetivo común que es el desarrollo infantil de la primera infancia. Lo anterior sugiere que las acciones disciplinares en Fonoaudiología se dinamicen en estructuras vinculantes con las maestras y los contextos reales en los que viven los niños y las niñas. Lo anterior implica no sólo considerar los aspectos formales del lenguaje sino que también abordar las interacciones y las dimensiones discursivas.

De manera particular, lo expresado por las maestras demuestra que el acompañamiento fonoaudiológico les ha permitido reconocer múltiples lenguajes con los que los niños se expresan, lo que aumentó sus posibilidades para comprenderlos y seguir a su desarrollo. Adicionalmente, han acrecentado el valor que le otorgan al juego como experiencia que promueve la expresión de intenciones, deseos, emociones y sentimientos.

Asimismo, las maestras refieren que han movilizado saberes que le han permitido la adquisición de herramientas en pro del desarrollo del lenguaje y la construcción de mundos simbólicos. Las actividades rectoras para la primera infancia -arte, juego, exploración del medio y literatura- también han enriquecido su actuación pedagógica con la disposición de generar actividades grupales que promuevan interacciones entre los pequeños $y$ pequeñas que los enfrente a situaciones que les exija hacer uso de distintos recursos comunicativos, adoptando expresiones más asertivas.

Por otra parte, señalaron que el acompañamiento fonoaudiológico les ha permitido ser conscientes que su disposición emocional es un elemento importante en la relación con las niñas y los niños, dado que ellos realizan lecturas constantes del mundo que les rodea, incluyendo las actitudes, comportamientos y expresiones no verbales de los adultos. También el acompañamiento fonoaudiológico les amplió la perspectiva sobre la observación, ya que puede ser una herramienta para realizar seguimiento a las niñas y los niños. Dicho seguimiento debe ir más allá de lo que ocurre en el entorno inmediato, lo que requiere abordar los diferentes contextos e historias de vida de los niños y 
niñas.

Es importante destacar que las maestras mencionan que el acompañamiento además ha propiciado la reflexión sobre el concepto de participación. Esta reflexión considera una mirada sobre el desarrollo del niño y no una perspectiva desde el mundo de los adultos. Estas reflexiones reconocen la importancia de los diversos actores de los entornos educativos en la eliminación de las condiciones de vulnerabilidad y la configuración de oportunidades para el desarrollo de todos los niños y las niñas. Con ello, las maestras mencionan la importancia de consolidar redes entre el acompañamiento fonoaudiológico, la familia y las maestras del CDI, pues la coordinación de las acciones que se realiza desde cada ámbito potencia el desarrollo infantil.

Las consideraciones anteriores, permiten sugerir la necesidad de dinamizar la Fonoaudiología en función del reconocimiento y la valoración de los saberes de los beneficiarios disponiendo acompañamientos o intervenciones más significativas. Lo anterior, requiere de la disposición de una acción situada, pues la comunicación, el lenguaje y la inclusión solo adquieren sentido en los contextos reales en los que se desenvuelve cada sujeto.

Finalmente, la investigación señala la importancia de la presencia del fonoaudiológico en la atención a la primera infancia. Esto porque el objeto disciplinar se constituye como un pilar para el desarrollo infantil. Sin embargo, esta afirmación, también invita a consolidar nuevas miradas al interior de quienes ejercen la disciplina desde la investigación y la acción cotidiana.
Es necesario que nuevas investigaciones amplíen las acciones de acompañamiento fonoaudiológico en la infancia considerando los contextos reales y los mecanismos de análisis discursivo. Igualmente, es necesaria la acción interdisciplinar con otros actores interesados en proteger a los niños y las niñas. Por ello, es necesario seguir sistematizando experiencias con enfoques diferentes para disponer del saber científico-técnico de la Fonoaudiología a la luz de los contextos reales.

\section{Referencias}

Camargo, M., Reyes, Y., \& Suárez, D. (2014a). El sentido de la Educación inicial (Documento No. 20). Bogotá, Colombia: Ministerio de Educación Nacional. Recuperado de http://www.deceroasiempre.gov.co/Prensa/CDocumentaci onDocs/Documento-N20-sentido-educacion-inicial.pdf

Camargo, M., Reyes, Y., \& Suárez, D. (2014b). La literatura en la educación inicial (Documento No. 23). Bogotá, Colombia: Ministerio de Educación Nacional. Recuperado de

http://www.deceroasiempre.gov.co/Prensa/CDocumentaci onDocs/Documento-N20-sentido-educacion-inicial.pdf

Caro, L. Á., Chávez, L. M., \& Vásquez, O. (2014). Modalidades y condiciones de calidad para la educación inicial (Guía orientadora No. 50). Bogotá, Colombia: Gobierno de Colombia. Recuperado de https://www.mineducacion.gov.co/1621/articles341863_archivo_pdf.pdf

Comisión Intersectorial para la Atención de la Primera Infancia. (2012a). Atención Integral: Prosperidad para la Primera Infancia (p. 32). Colombia: Gobierno de Colombia. Comisión Intersectorial para la Atención de la Primera Infancia. (2012b). Lineamiento técnico de formación y acompañamiento a familias de niños y niñas en la primera infancia (p. 11). Colombia: Gobierno de Colombia.

de la Fuente, C. (2012). Anexo Técnico para Orientar la prestación de servicios en un Centro de Desarrollo Infantil. Bogotá, Colombia: Instituto Colombiano de Bienestar 
Familiar. Recuperado de https://docplayer.es/8459426Anexo-tecnico-para-orientar-la-prestacion-de-servicios-encentros-de-desarrollo-infantil-modalidad-institucionaldireccion-de-primera-infancia.html

DeAnda, S., Bosch, L., Poulin-Dubois, D., Zesiger, P., \& Friend, M. (2016). The Language Exposure Assessment Tool: Quantifying Language Exposure in Infants and Children. Journal of Speech, Language, and Hearing Research, 59(6), 1346-1356. https://doi.org/10.1044/2016_JSLHR-L-15-0234

Duarte, J. D. (2011). Consideraciones finales. Revista Latinoamericana de Ciencias Sociales, Niñez y Juventud, 9(2), 145-160.

Instituto Colombiano de Bienestar Familiar. (2012). Diseño e implementación del Plan Operativo para la Atención Integral - POAl (Guía orientadora No. 1). Bogotá, Colombia. Recuperado de https://www.icbf.gov.co/sites/default/files/anexo_2._dise no_e_implementacion_plan_operativo.pdf

Lansdown, G. (2005). ¿Me haces caso?: el derecho de los niños pequeños a participar en las decisiones que los afectan. La Haya (Países Bajos): Bernard van Leer Foundation. Recuperado de https://www.observatoriodelainfancia.es/oia/esp/docume ntos_ficha.aspx?id=1930

Lizarazo, A. M., \& Fique, D. (2013). Tendencias de investigación fonoaudiológica: encuentros nacionales de investigación en Fonoaudiología. Areté, 13(1), 138-151.

López, M., \& Tobón, M. (2015). Fiesta de la lectura, promoción de la lectura y desarrollo de lenguajes y expresiones artísticas en la primera infancia. Guía 2. Bogotá, Colombia: Editorial Nomos.

Martínez, L. M. (2007). Breve mirada a la investigación en el campo de la fonoaudiología en Colombia. Areté, 7(1), 916.

Montes, M., Quessep, I., Redondo, L., \& Lastre, K. (2017). Tendencias De La Investigación En Fonoaudiología: El Caso De La Universidad De Sucre. Revista Colombiana de Rehabilitación, 14(1), 98-108.
Muñoz-Caicedo, A., Zapata-Ossa, H., Pérez-Tenorio, L. M., \& Illera, S. (2012). Prevalencia institucional de alteraciones en lenguaje y audición en niños entre 4 a 5 años. Revista de la Facultad de Ciencias de la Salud de la Universidad del Cauca, 14(4), 13-19.

Organización Mundial de la Salud. (2009). Subsanar las desigualdades en una generación. Buenos Aires: Organización Mundial de la Salud. Recuperado de http://www.who.int/social_determinants/final_report/clos ethegap_how/es/

Pava-Ripoll, N., Payán, C., \& Reyes, A. (2011). Aportes desde la investigación formativa a la producción en Fonoaudiología: el caso de una universidad colombiana. Revista da Sociedade Brasileira de Fonoaudiologia, 16(4), 377-383. https://doi.org/10.1590/S151680342011000400003

Pianesi, F., Scorpecci, A., Giannantonio, S., Micardi, M., Resca, A., \& Marsella, P. (2016). Prelingual auditoryperceptual skills as indicators of initial oral language development in deaf children with cochlear implants. International Journal of Pediatric Otorhinolaryngology, 82, 58-63. https://doi.org/10.1016/j.ijporl.2015.12.024

Puche, R., Orozco, M., Orozco, B., \& Correa, M. (Eds.). (2009). Desarrollo infantil y competencias en la primera

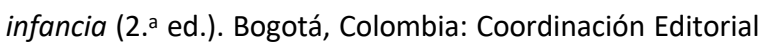
Unidad de Educación para la Primera Infancia, Ministerio de Educación Nacional. Recuperado de https://www.mineducacion.gov.co/primerainfancia/1739/ articles-178053_archivo_PDF_libro_desarrolloinfantil.pdf

Rubin, H. J., \& Rubin, I. S. (1995). Qualitative Interviewing:

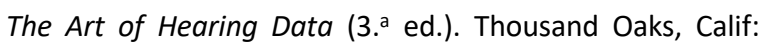
SAGE Publications, Inc.

Sen, A. (2000). Desarrollo y libertad. (E. Rabasco \& L. Toharia, Trads.). Barcelona: Planeta.

Taylor, S. J., \& Bogdan, R. (1994). Introducción a los métodos cualitativos de investigación: la búsqueda de significados (Vol. 1). Barcelona, España: Paidós. 
Téllez, S., Camargo, M., Reyes, Y., \& Suárez, D. (2014). Seguimiento al desarrollo integral de las niñas y los niños en la educación inicial (Documento No. 25). Bogotá, Colombia: Ministerio de Educación Nacional. Recuperado de

http://www.deceroasiempre.gov.co/Prensa/CDocumentaci onDocs/Documento-N25-Seguimiento-desarrollo-integralni\%C3\%B1as-ni\%C3\%B1os-educacion-inicial.pdf

Yáñez, V. (2013). Revisión epistémica a la constitución del estatuto disciplinar del Trabajo Social: mediaciones de sentido entre el pensamiento reflexivo y la acción creadora. Eleuthera, 8, 232-251. 